\title{
An investigation of the nature of termination of pregnancy counselling within the current system of licensed facilities
}

Shelley Kirk ${ }^{1}$, Liz Beddoe ${ }^{2}$ and Shirley Ann Chinnery ${ }^{2}$

\begin{abstract}
INTRODUCTION: Termination of pregnancy (ToP) service delivery in Aotearoa New Zealand occurs within a multi-dimensional system which is influenced and shaped by various philosophical, political and economic discourses, and is comprised of interconnected components. One component is the provision of counselling for women seeking a termination of pregnancy. This study aimed to explore how service managers and social work practitioners perceived how ToP services, particularly the counselling component therein, were being delivered nationally.

METHODS: A concurrent, multi-level, mixed-methods research design was employed in the study. Two purposively selected sample groups comprising: 1) service managers responsible for the oversight of ToP service delivery; and 2) ToP counselling practitioners were recruited from 19 District Health Boards (DHBs) across Aotearoa New Zealand. Service managers (20) participated in interviews with a focus on capturing information about operational systems that supported or hindered the delivery of ToP and counselling services, while 26 social work and counselling practitioners participated in an electronic survey questionnaire. Qualitative data were thematically analysed and quantitative data were descriptively analysed using descriptive statistics.
\end{abstract}

FINDINGS: Results from this mixed-methods study were integrated at the level of interpretation and linkage between the methods showed that practice within ToP licensed facilities varied markedly. Specifically, nine practice and systemic variations were identified that had implications for women receiving ToP services. Existing variations across licensed facilities were signalled as disconnects between components of the service delivery system.

CONCLUSIONS: Recommendations that address variations and systemic disconnects are offered to the New Zealand Abortion Supervisory Committee and Ministry of Health. Further research is suggested to obtain the perspective of service users as this was one limitation of this small exploratory study.

KEYWORDS: Abortion; termination of pregnancy; counselling; social work

Elective abortion is one of the most common medical procedures experienced by women in Aotearoa New Zealand. The Abortion
Supervisory Committee (ASC) states

"[i]nduced abortion ... affects about one third of women in their lifetime" (ASC, 2009, p. 1).
${ }^{1}$ Lakes District Health Board

${ }^{2}$ University of Auckland
AOTEAROA

NEW ZEALAND SOCIAL WORK 30(3), 31-44.

CORRESPONDENCE TO:

Shelley Kirk

Shelley.Kirk@

lakesdhb.govt.nz 
The history of elective abortion in Aotearoa New Zealand and associated moral and political discourses have been well documented by Sparrow (2010) and Richdale (2010) and offer background and impetus to the passing of the Contraception, Sterilisation and Abortion (CSA) Act (1977) and subsequent appointment of the Abortion Supervisory committee in 1978 to oversee provision of services under the Act and report to parliament. While a full account is precluded by space considerations, a brief outline of the current legal situation is necessary at this juncture.

\section{Legislation}

The relevant New Zealand legislation, the CSA Act (1977) and the Crimes Act (1961), have largely remained unchanged since their inception and have provided the legal framework through which current abortion services are delivered. In the context of this study, elective abortions rather than spontaneous abortion (miscarriage), will be henceforth referred to as termination of pregnancy (ToP). The Crimes Act (1961) specifies that abortion is unlawful (Crimes Act (1961), ss182, 183, 186, 187 \& 187a) unless it occurs within the scope of the CSA Act (1977); and provides clear criteria for what constitutes a lawful abortion:

- $\quad$ The pregnancy is not more than 20 weeks' duration;

- " $[\mathrm{T}]$ he continuance of the pregnancy would result in serious danger (not being danger normally attendant upon childbirth) to the life, or to the physical or mental health, of the woman or girl" (Crimes Act (1961) s187a);

- The unborn child is at risk of serious abnormality;

- The pregnancy is a result of incest;

- The pregnancy is a result of sexual assault that is an offence under the Crimes Act (1961).

The Crimes Act (1961) also provides a framework that prescribes the licensing of abortion facilities, how medical and supportive services are to be delivered, and by whom. Their functions are regulated separately by the ASC which operates under the funding arm of the Ministry of Justice and the legal mandate of the CSA Act (1977). Of the 46 sections within the CSA Act (1977) only ss 31 and 35) directly pertain to the provision of counselling. Furthermore, the recording and reporting requirements outlined in the Act are only concerned with the medical/ surgical performance of abortions.

\section{The provision of counselling}

The provision of counselling services to women seeking an abortion is the focus of this study. Silva, Ashton, and McNeill (2011) note that, "[b]y law, all women, must be offered counselling throughout the process, although the law does not specify that women must receive counselling" (p. 20). This necessitates that a counselling session must be made available to women as part of the service delivery of abortions. The ASC is charged with the responsibility to ensure that "sufficient and adequate facilities are available throughout New Zealand for counselling women who may seek advice in relation to abortion" (ASC, 1998, p. 12).

The aim of the study was to investigate the current operational systems and processes and counselling practices that underpin and support the termination of pregnancy within licensed abortion facilities as detailed on the New Zealand Abortion Services website (www.abortionservices.org.nz). The term "systems" pertains to operational aspects e.g., funding pathways, reporting and documentation processes (statistical data capture, case notes, file storage and referral reports); and supportive aspects_training, supervision, qualification requirements for those providing counselling. The overarching aim was further specified by two sub-questions:

- What features of the current systems of ToP counselling service delivery support or hinder consistent and quality national services? 
- Who is providing the ToP counselling service and what is their experience of the current system within which they operate?

The findings of this small exploratory study are explored with reference to integrated care pathways, which should be client centred and developed from the involvement/ integration of all key stakeholders concerned with services delivery. Variances in both practice and processes in services suggest disconnects, which impact on the quality of care. The aim of this article is to provide an overview of this small-scale, descriptive study and its combined findings.

\section{Literature review}

A literature review was undertaken which included qualitative and quantitative studies, case reports, surveys, reports from New Zealand government and other organisations, technical reports and crosssectional studies. There is considerable literature, both national and international, regarding ToP, however, much of it is aligned to the fields of medicine and nursing rather than social work or counselling.

Aotearoa New Zealand literature that directly related to ToP counselling services was found to be extremely sparse and dated. Relevant material located consisted: of four articles (Beddoe \& Weaver, 1988; Hunton \& Spicer, 1979; Leask, 2014; Silva et al., 2011); two reports (Federation of Women's Health Councils, 1992; Nicol, 1987); two ToP procedural guidelines (ASC, 1998; and ASC, 2009); and associated legislation (CSA Act, 1977; Crimes Act, 1961). Other material of New Zealand origin related to ToP service delivery in general, funding and contracting processes, and the historical context of the development of Aotearoa New Zealand legislation and ToP services. The search was expanded to encompass managerial and operational themes such as workforce development, care pathways, models of care, quality improvement, integrated care and clinical governance to enhance understanding regarding ToP service delivery. International sources (34) resulted from the foregoing search and these related to ToP counselling in Australia, UK and USA. The located sources were derived from a variety of professional and academic disciplines, for example, psychology, mental health, sociology, medical, nursing, and women's studies. The literature search was expanded upon because the initial search generated only one source that described all systemic components related to the delivery of ToP services (Family Planning Association, 2015).

Debates about who should provide ToP counselling were highlighted in the international literature. Different types of counselling were identified within this literature as important and comprised: information giving; implications relating to decisions; support and therapeutic counselling (Lee, 2011). Variations in counselling were also found and these appeared to differ in accordance with the practitioner's role, for instance, provided by a range of health practitioners and lay counsellors (Illsley \& Hall, 1976). Notably, the literature indicated that many of these practitioners were not specifically trained to deliver ToP counselling (Brien \& Fairburn, 2003). Other commentators (Needle \& Walker, 2007; Federation of Women's Health Councils, 1992) firmly placed health social workers as being "uniquely situated within the medical context ..." and as having the ability/skills to "... traverse the dilemma-filled processes of decision-making, termination and bereavement" of ToP counselling (McCoyd, 2010, p. 147).

Social work practice is underscored by principles of human rights and social justice (International Federation of Social Workers, 2015). Central to practice is the view of the client in context-the perspective that each individual exists within a complex system of relationships and interactions that are environmental, socio-political, interpersonal and intrapersonal, and influenced by a variety of values, beliefs and characteristics of this multi-dimensional system within 
any given circumstance. The social work role in ToP counselling is to provide a client-centred therapeutic approach to assist women to negotiate the psycho-social aspects of decision-making regarding an "unwanted" pregnancy. Counselling assists women to explore options, rationalise and minimise emotional discomfort, validate their choice and enables them to determine their own future. This also applies to those situations where ToP may be considered due to the pregnant woman's health being at risk or where there is a diagnosis of foetal abnormality. From a social work perspective, ToP counselling is a process comprising exploration and normalisation of the client context, supporting the client's choice, providing information and preparation around the client's choice (whether this be ToP or not), and, additionally, attending from a strengths-based perspective to any contextual issues of social risk, for example, family violence, addictions and planning for future well-being (Ely, Dulmas, \& Akers, 2010).

The CSA (1977) clearly states that ideally social workers should provide counselling for ToPs, however, the only reference to specific qualification requirements is found in the Standards of Practice for the Provision of Counselling (ASC, 1998) and these do not reflect the contemporary education and regulation of social workers, thus are out of date.

The lack of literature reporting relevant research conducted in Aotearoa New Zealand, and the lack of any recent information about the provision of counselling was the impetus for the study reported in this article.

\section{Methodology}

A mixed methods concurrent design was employed to obtain a greater understanding of ToP service delivery and counselling, capitalising on the strengths of both singular methodologies by combining inductive (qualitative) with deductive (quantitative) methods. The study employed a purposive sampling strategy of two, multi-level groups within the same organisation, namely 20 ToP service managers and 26 counselling practitioners (Onwuegbuzie \& Leech, 2007).

\section{Ethics approval}

The study was undertaken for the fulfilment of a Master of Social Work degree and ethics approval was sought from the University of Auckland Human Participants Ethics Committee (ref: 012336). Additionally, Locality Ethics Approval was sought from several DHBs upon their request.

\section{Participant recruitment}

Participants were sought from two predefined populations representing different levels within the same organisation: 1 ) service managers identified/elected by individual DHBs as having oversight of the delivery of ToP services and who could offer an operational viewpoint; and 2) social workers/lay counsellors providing the counselling who could offer a practice viewpoint. The aim was to be inclusive of all potential participants: 19 service managers and an estimated population of between 50-100 counselling practitioners.

Recruitment occurred via a formal letter of invitation (including research participant information and consent forms) sent to the Chief Executive Officers of each licensed facility (19 District Health Boards and three private abortion clinics) requesting consent for service managers and ToP counselling practitioners to participate in the study. Service managers were then contacted directly to arrange interviews; additionally, they were sent a copy of the interview questions and an electronic survey link to be distributed to ToP counselling practitioners. Participant information regarding the research introduced the electronic survey and continuation to complete the survey was deemed as providing consent. 


\section{Data collection and analysis}

A total of 20 semi-structured interviews with service managers from 14 DHBs were conducted in 2016. Two interview processes were used (face-to-face and via telephone) depending on participant preference and geographical location. The interviews were audio-taped for the purpose of transcribing. All interviews were designated a random numerical code to ensure anonymity of both DHB and individual participants. An anonymous electronic survey host on SurveyMonkey enabled the participation of 26 social workers/counsellors in the study.

Each data set was analysed by the first author according to its protocol and subsequently combined at the level of interpretation. For instance, qualitative data were thematically analysed in accordance with Braun and Clarke's thematic analysis approach $(2006,2014)$. This involved themes being identified by systematically coding all data with the identification of repetition, similarities, difference and significance within the content of coded extracts (Braun \& Clarke, 2014). Thematic mapping was utilised to organise themes, which coalesced around systemic aspects of the ToP services. Quantitative data were descriptively analysed (Onwuegbuzie, Slate, Leech, \& Collins, 2007).

The data from the two separate methods were analysed separately and then merged in a side-by-side approach (Creswell, 2014). For example, one area of convergence within the data was that the ToP counselling workforce is primarily qualified and registered female social workers who are employed to undertake this area of work. Integration of data occurred at an analytical level to create meta-inferences (Leech \& Onwuegbuzie, 2007); and are presented in the Findings section.

\section{Findings}

The qualitative findings from interviews were organised and presented under five (researcher derived) broad themes and related sub-themes. These are: funding, reporting, delivery, staff profile and training. Quantitative findings from survey participants were used to describe the demographics of the ToP counselling workforce.

\section{Funding}

The funding theme was aimed to describe data about funding pathways: how much funding was allocated and utilised within DHBs for ToP services; whether there were variations across DHBs; how contracts linked to funding; and how ToP counselling fitted into the funding stream. Four types of funding were identified in the textual narrative of the interview respondents.

Of the 20 managers interviewed, 50\% noted that the Ministry of Health (MoH) funds for DHBs for ToP services were via price volume schedule as part of the population-based funding system: “. . a planned volume is set and reviewed by the DHB each year as part of setting the price volume schedule" (Manager, small DHB). Of others, 15\% identified that part of the ToP services was contracted to or from other DHBs via "inter-district flow": an exchange of funds between DHBs relating to sub-contracted services; or (10\%) via a "fees-for-service" process where services were funded only by the number delivered. Data indicated that not all funding processes were well understood by participants. Variances in understanding may have been related to whether the particular role was operational management or clinical leadership, or due to the nature of how ToP services were designed. What was clearly highlighted is the alignment of funding to contracts $(\mathrm{MoH}$ service specifications) which outlined service delivery and accountability mechanisms.

The provision of ToP counselling was viewed by half the participants as an element of general social work services delivery, for example, "...it's just part of their general work and I've never been given an increase in resources to cover that..." (Manager 1, 
large DHB). The generic social work funding was described as a normal mechanism for ToP counselling in most DHBs; however, there was also a separation of funding dependent on the type of ToP services being provided. Terminations of foetal abnormalities were funded differently than early first or second trimester terminations: "....if they're a foetal termination then we fund it out of our secondary service bucket" (Manager, small DHB).

\section{Reporting}

Reporting captured data regarding how participants understood reporting mechanisms and documentation relating to ToP counselling and how these are supported within service delivery. Statistical data regarding individual client contacts were of particular importance as these are directly aligned to funding matters: price volume schedules, service specifications and purchase unit codes and part of reporting processes to $\mathrm{MoH}$. However, 35\% of participants indicated that statistical data capture specific to ToP counselling was poorly understood and not particularly visible with the general ToP services data capture.

Variances concerning documentation were noted in the responses of most $(86 \%)$ of the interview participants, although they acknowledged some type of documentation, usually in the form of counselling reports. Almost half of the survey respondents offered further clarity, stating they documented counselling notes in either the patient clinical file $(48 \%)$ or a separate complementary file $(40.74 \%)$; and that information was conveyed to the ToP multi-disciplinary team either verbally or via a written report. The ASC counselling standards outline that ToP counsellors should "observe, record and accurately report on sessions (verbally and in writing) what is presented by the client" (1998, p. 10).

Client information confidentiality, access to, and storage of, information also differed across survey participants. Most (73.07\%) stated they had a secure process for storing client information separate from, or in a sealed section of, the main patient file. Almost half (46.15\%) reported that only staff providing ToP services had access to the client information but noted this was also dependent on other staff following organisational policies. Variations in documentation processes may be attributed to how individual DHBs manage their clinical information and how information is transferred between DHBs.

\section{Delivery}

Counselling is only one of the multiple components that are designed to interlink to form a process for how ToP services are to be delivered. Delivery, as a theme, was utilised to encapsulate concepts around how ToP counselling services were provided across the 19 DHBs.

Over half (65\%) of interview participants indicated they provided ToP services on site, the remainder sub-contracted these from other DHBs. Most (86\%) stated they subcontracted second-trimester ToP services from other DHBs or private providers. Over half $(65 \%)$ provided some limited services for foetal abnormality ToP, and those with further advanced gestation were again subcontracted out. There were notable variances across the country with larger DHBs providing the whole range of services while smaller DHBs sub-contracted out part or all of the ToP service. During the course of this study, one DHB separated into two districts. The reasons offered for out-sourcing ToP services were: lack of medical facilities; shortage of surgical personnel willing to provide this service; and diagnostic delays.

All DHBs provided pre-decision and posttermination counselling services. Those providing the medical component of ToP services also provided counselling on the day of surgery. In contrast, all survey participants reported providing pre-decision counselling, $59.26 \%$ provided on the day of 
ToP counselling, and $85.19 \%$ provided postToP counselling. No participants reported restrictions within $\mathrm{DHB}$ policies on the number of pre-decision counselling sessions a woman could have, although the general consensus was that between one and three sessions were acceptable. The ASC Abortion Services in New Zealand website suggests post-ToP counselling should not exceed six sessions.

Research by Whitehead and Fanslow (2005) indicates that family violence for Aotearoa New Zealand women seeking terminations is common. Routine FV screening of women over 16 years to ensure early identification and assistance is a requirement under the Ministry of Health Family Violence Intervention Programme (2002). Survey participants $(96.15 \%)$ responded that they routinely screened for family violence during ToP counselling. Additionally, survey participants stated that there were enough $(55.55 \%)$ and generally accessible $(69.96 \%)$ services in their area to respond to family violence disclosures. Most (62.96\%) felt these services were responsive to family violence referrals but were not necessarily available 24 hours and seven days per week - the exception being Women's Refuge.

\section{Staff profile}

Staff profile describes the staff providing ToP counselling and included concepts regarding: profession; professional requirements; recruitment/employment of counselling staff; and whether the practitioner's role was a designated position.

Interview participants reported a national total of 45 practitioners providing ToP counselling, with a range from one to five employed depending on the size of the DHB. Survey participants represented (58\%) of this workforce. Demographic details obtained via survey participants described the counselling workforce as $100 \%$ female, aged between 21 and $60+$ years with the majority of the workforce aged between 40 and 59 years, and largely European. Of those aged
40 years or older, $42 \%$ claimed an advanced level of practice experience (seven or more years) in the field of ToP counselling. Those who identified as Māori were $7.42 \%$, lower than the national average of $15.6 \%$ (Statistics New Zealand, 2015, p. 1). ToP statistics for 2013 indicate Māori were the second-highest population group nationally $(24.57 \%)$ and Asian the third highest (17.08\%)—neither of these population groups are adequately represented in the ToP counselling workforce (ASC, 2015).

Interview participants (70\%) reported that their counselling staff held either a counselling or social work qualification. Survey participants reported $(84.61 \%)$ as holding social work qualifications with the remainder holding a counselling qualification. Both participant groups reported that counsellors held professional memberships with either the Aotearoa New Zealand Association of Social Workers or the New Zealand Association of Counsellors (NZAC). Most interview participants $(76 \%)$ reported their staff were registered regardless of their profession. In contrast, $92.31 \%$ of survey participants reported they were registered practitioners (either social work or counselling) and 100\% reported holding a current annual practising certificate. At present, NZAC does not have a statutory registration process; an application for full membership process is carried out as a form of self-regulation.

Interview participants $(76 \%)$ reported recruitment to ToP counselling occurred via staff already employed, who were interested in this field of practice and had experience. There was variation in how experience was determined: both length of service and experience in health-related areas were identified. Additionally, $85 \%$ of interview participants stated ToP counselling was absorbed into other aspects of social workers' daily workload. "We don't have any allocated FTE [staff time] specific to it - it is all done within generic FTE" (Manager, small DHB). Only four DHBs represented by $30 \%$ of interview participants reported 
having FTE designated specifically to ToP counsellors.

\section{Training and support}

Training, as a theme, describes concepts regarding ToP counselling training, professional development, including preparation and support for entry to this field of practice.

Four interview participants expressed concern about the lack of a national training programme, however, there was a general consensus that $\mathrm{ToP}$ counselling training occurred internally within individual DHBs and was conducted via three primary methods: learning information; use of resources utilised within ToP counselling sessions; and via a person-to-person approach. In comparison, $88.89 \%$ of survey participants described their training as "on-the-job" or "in-house" ranging from some form of orientation/induction, coaching/mentoring by a more experienced practitioners to more intensive training from an experienced practitioner. Intensive training included reading associated material, attending peer presentations, role playing and observations of live ToP counselling sessions. Only $29.63 \%$ of survey participants stated they had been able to attend training days organised on a regional basis or a national conference. There was no indication of consistency of training method across DHBs which raises questions about how this may impact on the quality of ToP counselling service delivery.

Support to counsellors was described by interview participants as provided in clinical supervision (75\%), multi-disciplinary meetings, peer debriefing post ToP clinics, and regional meetings (bi-annually). Continuing professional development was presumed to be included for discussion within generic annual appraisals and professional development planning. In contrast, survey participants $(95.15 \%)$ stated they receive regular clinical supervision in addition to supports as stated above.
In relation to future developments, survey participants were asked whether nationally approved ToP counselling training, standardisation of documentation processes and nationally agreed ToP counselling resources might enhance ToP counselling services. While $88.46 \%$ agreed that it would be beneficial to have a nationally recognised ToP training programme, there were some reservations about this being too prescribed. Regarding a nationally standardised documentation process, $73.01 \%$ felt this would be beneficial and $73.01 \%$ were in favour of having access to a nationally approved set of resources that would be utilised within counselling sessions.

\section{Discussion}

As the focus of this study was quite pragmatic-responding to the need to understand how counselling services were perceived by managers and practitionersnew knowledge derived from this study is discussed through the lens of the integrated care pathway. The integrated pathway is defined by Johnson as an "... amalgam of all the anticipated elements of case and treatment of all members of the multidisciplinary team, for a patient or client of a particular case-type or grouping ..." (Johnson, 1997, p. 16). Integrated care pathways are: client centred and developed from the involvement/integration of all key stakeholders concerned with services delivery; a mechanism to facilitate best practice; for ensuring continuous quality improvement. Problematic variations within the data were identified as disconnects and implications for ToP counselling service delivery were highlighted. For example, the law (CSA Act, 1977) stipulates that GPs and certifying consultants must offer counselling, it does not include nurses. Nurses employed by GP services and family planning centres are assumed to be potential referrers for ToP counselling clients. In contrast, the Standards of Care (ASC, 2009, standard 27, p. 13) requires those involved "caring for a woman requesting abortion must advise a woman of her right to seek counselling and 
facilitate her referral to a suitably trained and credentialed professional whose counselling practice meets the standards of the ASC. This service must be free and easily accessible".

Standard 29 (p.13) states that a counsellor

“a) hold a relevant qualification or have equivalent training in abortion counselling.

b) be registered members of their profession, e.g., counselling or social work. c) be doing regular pregnancy counselling for women considering abortion, d) have supervision and peer review". This signals a disconnect between law and practice, if an appropriate qualification is not held.

The ToP care pathway differs from most other health care pathways in that clients must meet both medical and legal requirements to undertake this procedure. The requirement that counselling must be offered and available (CSA Act, 1977), acknowledges and legitimates that there is a strong psycho-social element associated to ToP service delivery. While not all women seeking termination require counselling (Marie Stopes International, 2006), it is the psycho-social aspects associated with ToPs that give rise to the need for counselling and the politics surrounding abortion (Needle \& Walker, 2007).

One disconnect that impacts on ToP service delivery is the need to meet the legal requirement of lawful access to services on the grounds of serious danger to the women's physical or mental health should the pregnancy continue (Crimes Act, 1961, s187a). This perpetuates the discourse that mental illness is directly related to and an outcome of procuring a ToP, rather than psychological distress related to an unexpected/unplanned or unwanted pregnancy occurring within the context of the individual's personal situation. This is a significant issue given that, in $2014,97.3 \%$ of abortions performed in Aotearoa New Zealand were for the above-stated grounds (ASC, 2015, p. 20). Locating abortion within the criminal code has implications for coordinated policy and service development.
Legislation should ideally reflect the reality of the context in which abortion occurs. It is important to note is that since the completion of this study, Andrew Little (Minister of Justice) requested the Law Commission review the criminal aspect of Aotearoa New Zealand abortion law. Part of the review is to consider whether abortion legislation might be better aligned to health rather than the Crimes Act (1961).

\section{Workforce implications}

In common with other OCED countries, Aotearoa New Zealand is faced with an aging workforce- those of retirement age (65+ years) are predicted to increase to $23 \%$ by $2036(\mathrm{MoH}, 2014)$. Survey data indicate that $70.37 \%$ of the ToP counselling workforce are aged between $40-59$ years and are also those who have the most experience in this particular field of practice. The availability of a suitable ToP workforce dictates how care pathways are developed and may have impacts on accessibility of counselling for women seeking ToPs. Study findings suggest that there is no workforce development plan in relation to recruiting either medical or counselling staff to this field. This signals a need to address future workforce development and ToP service delivery design. Such a review should include the incorporation of consistent and accessible ToP counselling.

DHBs have the scope to develop ToP services to meet their local/regional needs (Ministry of Justice, 2014) and counselling variations are largely influenced by whether the medical component occurs on site or is outsourced. Those who provide the whole service are likely to provide to a large population or catchment and are also likely to have a designated resource for ToP counselling with time allocated to practitioners or a specific allocated role. There are few indications from the data that any funding is specifically captured for ToP counselling services. Variances concerning allocated resource to ToP counselling identified in this study indicate that this 
specialist role has not been adequately scoped and may be poorly integrated into the ToP care pathway.

The CSA Act (1977) stipulates that GPs / certifying consultants must offer counselling to women seeking a service; and that referrals are to be a "suitably trained and credentialed counsellor" (ASC, 2009, standards 27-29). This study reflects that it is largely qualified, registered health social workers providing the ToP counselling service in compliance with the law (CSA Act, 1977); and who are already employed in related care pathways e.g., obstetrics, women's medical services. There is still a need, however, to differentiate between the different types of counselling provided by different disciplines in relation to ToP care pathway.

While other health professionals/lay counsellors may be able to identify concerns and provide information about ToP procedures, social workers are also engaged with non-procedural counselling, for example, exploration of certainty, emotions, values and beliefs regarding their decision; identifying support; attending to contextual sources of anxiety, and managing safety and risk issues that might increase the clients' likelihood of poor coping. Data indicated that ToP counsellors excelled at routine enquiry regarding family violence; and participants responded positively regarding general availability, accessibility and responsiveness of family violence support services. Knowledge of these services is of paramount importance in successful referral.

\section{Credentials and professional development}

A second disconnect was identified in relation to credentialing-recruitment, orientation, training and continuing professional development across health disciplines in relation to ToP services. The $\mathrm{MoH}$ has a process that assigns clinical responsibilities to health practitioners based on training, qualifications and experience with the goal of ensuring patient safety $(\mathrm{MoH}, 2002)$ and which is supported by national regulatory bodies and professional associations (Health Practitioners Competency Assurance Act, 2003; the Social Workers Registration Act, 2003; New Zealand Association of Counsellors). The application of this process, however, is not consistent across all health disciplines in relation to ToP services. There are expectations within the Standards of Care (ASC, 2009) for both nursing and medical practitioners to undertake specialist training and engage in continuing professional development. In contrast, this study identified national variations in relation to role orientation and training being reliant on the broad expertise of more experienced practitioners in ToP counselling. Both interview and survey participants expressed a concern about the lack of training specific to ToP counselling. There is a lack of guidance from the Standards of Practice (ASC, 1998) regarding CPD requirements and level of expertise within the field of ToP counselling.

ToP services are not widely and openly advertised and this is likely due to the contentious political and moral nature of abortion. The most common method of accessing information about services, outside of GPs and health clinics, is via the internet. Not all DHBs offer information that can be accessed by their websites. This method is also a difficulty for those who live rurally, have poor internet coverage or where literacy might be a barrier. There is a legal requirement that ToP counselling be offered; however, it is unclear whether this service is promoted in a manner that encourages engagement. Data indicate national variance regarding ToP counselling services information via pamphlets or DHB websites. Survey participants (62.96\%) reported that their information pamphlets were largely distributed via ToP services rather than at the point of referral. Further variations were reported regarding resources utilised to aid informed decision-making with the likely implication of inconsistent quality regarding 
written information/decision-making tools being used. Both of these concerns signal a need for some nationally approved and standardised counselling and information resources.

Documentation was considered an issue of importance in that it is the mechanism by which ToP service delivery maintains public visibility. Data capture regarding ToP services was linked significantly with reporting against $\mathrm{MoH}$ contracts and funding accountability; and ASC statistical data collection. While social workers are required to capture statistics in relation to face-to-face client contacts in respect of reporting against $\mathrm{MoH}$ contracts there is no requirement to differentiate a ToP counselling contact from any other client contacts that occur in the course of their work. ToP counselling is generally captured generically and is thus often undistinguishable as there is no mechanism that requires it to be reported to the $\mathrm{MoH}$ or ASC. This signals a significant component of the ToP care pathway remains unreported nationally and thus invisible.

Variations were also noted in the way individual DHBs utilise their allocated funding for ToP services and that it is dependent on availability of clinical expertise, equipment, and effective use of financial and staffing resources. The implication is that ToP care pathways may be planned, developed or improved without being understood well by all involved and without equal consideration given to all components of services delivery. How funding processes affect implementation of service delivery sits at a high managerial level and not necessarily well understood by the managing staff or staff delivering the service. The method of allocating funding for social work services may not truly reflect the range of services or adequately cover the cost of all aspects of services being delivered.

The contribution of this study is to illuminate disconnects noted in the data between reporting requirements, the funding of (and contracts related to) ToP counselling services, and the features of a well-articulated integrated care pathway. The researchers note that these disconnects undermine the delivery of this important component of the care pathway for ToP; and signal that this aspect is inadequately imbedded in care pathways for many DHBs.

\section{Limitations}

While this small study attempted to provide an exploratory overview of ToP counselling services in Aotearoa New Zealand, there is one glaring deficit that should be remedied in future research and that is the voice of service users - this was not possible to explore within the time available to complete the project. There is need to obtain the perspective of the women utilising the counselling service component of seeking a ToP for, without this, there is risk of delivering services that primarily meet the needs of the providers. It is suggested that further research be undertaken to obtain the viewpoints of women who are recipients of ToP services.

\section{Conclusions}

Care pathways describe the client journey in relation to a health event, whilst models of care are concerned with design-the underlying philosophies, principles and processes which support the delivery of the care pathway. "Abortion is more than just a medical or surgical procedure. The procedure occurs within the context of a women's life ..." (Gawinski, Bennett, Rousseau, \& Schaff, 2002, p. 440); and ideally, the training of practitioners in abortion care should be founded on a biopsychosocial model.

Clinical governance provides the framework under which models of care and care pathways are developed; and should: involve all levels of the organisation; be linked to legislation, government requirements and professional standards of practice; and include input from service 
users. It should also include systems and processes that support service delivery, notably shared accountability, management of risk, and quality improvement. Data from interview participants suggest that few DHBs had recently reviewed their ToP services.

The UK's Family Planning Association (2015) provides a best practice guideline that outlines core principles in commissioning ToP services. Some, but not all, of the principles have been adopted by ASC (2009). One of the Family Planning Association's (2015) recommendations is that workforce development and training, and specialist support services such as counselling should be incorporated in service specifications.

There are a number of variations identified within this study that signal disconnects between legislation, policies, practice and funding in relation to ToP counselling services. These are:

- $\quad$ Funded travel and access to services, as highlighted by the Nicol Report (1987), remain an issue for many.

- Legislation that is not reflective of the current context for women seeking ToPs (this is now being reviewed by the Law Commission).

- Current aging ToP workforce signalling the need for a national workforce development plan.

- Legislation and practice guidelines that require improved alignment.

- Inconsistent application of ASC guidelines in relation to provision of information to clients.

- Lack of national guidelines to ensure consistent documentation in relation to ToP counselling services.

- ToP counselling services not included in the national ToP reporting mechanisms.

- Unclear funding pathway for, and lack of inclusion in, ToP contracts regarding counselling services.

The variations described above represent a disconnect between the systemic components of ToP service delivery and those that directly relate to ToP counselling services. ToP services occur within an interconnected, multi-dimensional environment.

We conclude the article with some recommendations for improvement of the counselling services by addressing the systemic concerns identified.

\section{Recommendations}

- The MoH national travel assistance scheme be reviewed to enable all women seeking ToP services eligibility for funded travel.

- $\quad$ The CSA Act (1977) be reviewed to remove the stigma of mental illness and consider psychological distress as a legal requirement for eligibility to ToP services.

- $\quad$ The MoH commence a national workforce development plan in relation to all health disciplines engaged in the delivery of ToP services.

- $\quad$ The ASC and the MoH ensure that practice guidelines for all components of ToP service delivery are aligned to legislation and reflect best practice.

- The $\mathrm{MoH}$ prescribe equitable credentialing processes across all disciplines employed to deliver ToP services; and are reflected in ASC guidelines.

Counselling is an important component of ToP service delivery and the results of this study highlighted disconnects that impact on quality and availability. Solutions are offered in the following recommendations that:

- The ASC develop nationally approved set of resources that can be provided to clients and utilised in counselling.

- The ASC develop nationally approved guidelines for documentation processes in relation to ToP counselling services that have flexibility to reflect regional needs.

- $\quad$ The ASC develop a national reporting mechanism by which to audit efficacy 
and quality of ToP counselling services-ensuring continuous quality improvement and visibility of this component of ToP services.

- The MoH develop a funding pathway via a separate purchase unit code for ToP counselling.

While there are many positives about the Aotearoa New Zealand termination of pregnancy services (Silva et al., 2011), the earlier identified variations in service delivery suggest that there are some areas of improvement needed to ensure quality ToP services delivery, especially that of ToP counselling. Further research is needed to gain service user views and inform better systems for the induction, training and professional development of social workers providing this important service.

Postscript: At the time of acceptance of this article (October 30, 2018) The Law Commission had published the briefing paper Alternative ApproachestoAbortion Law (Law Commission, 2018) commissioned by Justice Minister Andrew Little in February 2018, which provides three alternative legal models for consideration.

- Model A proposes there would be no specific abortion legislation and the abortion provisions in the Crimes Act (1961) and the Contraception, Sterilisation, and Abortion Act (1977) would be repealed. There would be no statutory test and the decision to have an abortion would be made by a woman in consultation with a health practitioner.

- Model B proposes a statutory test in health legislation, rather than under the Crimes Act. This test would require the health practitioner who intends to perform the abortion to believe it is appropriate considering the woman's physical and mental health and wellbeing.

- Model C combines aspects of A and $B$ and focuses on gestation. For pregnancies of not more than 22 weeks' gestation, model A would apply. For those more than 22 weeks, a statutory test of $\mathrm{B}$ would be required.

All options would lead to the repeal of current grounds in the Crimes Act and the need for certifying consultants. Any legislative development will result in changes to the provision of services, including counselling and social work support.

\section{References}

Abortion Supervisory Committee. (1998). Standards of practice for the provision of abortion counselling. Wellington, NZ: Government Printer.

Abortion Supervisory Committee. (2009). Standards of care for women requesting induced abortion in New Zealand [Report of a Standards committee to the Abortion Supervisory Committee]. Wellington, NZ: Ministry of Justice.

Abortion Supervisory Committee. (2015). Report of the Abortion Supervisory Committee. Wellington, NZ: Ministry of Justice.

Beddoe, L., \& Weaver, A. (1988). Ten years on: Abortion counselling services in New Zealand. New Zealand Social Work, 12(3/4), 23-28.

Brien, J., \& Fairburn, I. (2003). Pregnancy and abortion counselling. London, United Kingdom: Routledge.

Braun, V., \& Clarke, V. (2006). Using thematic analysis in psychology. Qualitative Research in Psychology, 3(2), 77-101.

Braun, V., \& Clarke, V. (2014). Successful qualitative research. London, UK: Sage.

Contraception, Sterilisation and Abortion Act, 1977, No 112. Retrieved from http://www.legislation.govt.nz/public/1977

Creswell, J. W. (2014). Research design. Thousand Oaks, CA: Sage.

Crimes Act, 1961, No 43. Retrieved from http://www. legislation.govt.nz/act/public/1961

Ely, G. E., Dulmus, C. N., \& Akers, L. S. (2010). An examination of levels of patient satisfaction with their abortion counseling experience: A social work practice evaluation. Best Practices in Mental Health, 6(2), 103-114.

Family Planning Organisation. (2015). Decision-making support within the integrated care pathway for women considering or seeking abortion: Guidance for commissioners on improving access and outcomes for women. Retrieved from http://www.fpa.org.uk/decision making_support_abortion

Federation of Women's Health Councils. (1992). Abortion services and the health changes. Wellington, NZ: Ministry of Health.

Gawinski, B. A., Bennett, P. A., Rousseau, S. J., \& Schaff, E. (2002). A biopsychosocial model of training in abortion care. Families, Systems and Health, 20(4), 439-446.

Health Practitioners Competency Assurance Act, 2003, No 48. Retrieved from www.legislation.govt.nz/act/ public/2003/0048 
Hunton, R. B., \& Spicer, J. (1979). An evaluation of the counselling given to patients having a therapeutic abortion. Australian and New Zealand Journal of Obstetrics and Gynaecology, 19, 169-173.

Illsley, R., \& Hall, M. (1976). Psychosocial aspects of abortion. Bulletin of World Health Organisation, 53. United Kingdom: Blackwell.

International Federation of Social Workers. (2015). Global definition of the social work profession. Retrieved from http://www.ifse.org/policies/definition-of-social-work

Johnson, S. (Ed.). (1997). Pathways of care. Carlton, VIC: Blackwell Science.

Law Commission. (2018). Alternative approaches to abortion law: Ministerial briefing paper. October 262018. Wellington, New Zealand: Author. Retrieved from https:// www.lawcom.govt.nz/abortion

Leask, M. (2014). Constructing women as mentally troubled: The political and performative effects of psychological studies on abortion and mental health. Women's Studies Journal, 28(1), 74-82.

Lee, E. (2011). Pregnancy counselling in Britain: A review of literature. Psychology and Reproductive Choice. Retrieved from http://www.prochoiceforum,org.uk

McCoyd, J. L. M. (2010). Women in no man's land: The abortion debate in the USA and women terminating desired pregnancies due to foetal anomaly. British Journal of Social Work, 40, 133-153.

Marie Stopes International. (2006). What women want when faced with an unplanned pregnancy. (Websurvey Report), Melbourne, VIC: Author.

Ministry of Health. (2002). Family violence intervention guidelines: Child and partner abuse. Wellington, NZ: Author.

Ministry of Health. (2014). The role of Health Workforce New Zealand. Wellington, NZ: Author.

Ministry of Justice. (2014). Abortion services in New Zealand: DHB responsibilities. Retrieved from http://abortionservices.org.nz/ministry

Needle, R., \& Walker, L. E. (2007). Abortion counseling: A clinician's guide to psychology, legislation, politics and competency. New York, NY: Springer Publishing.

New Zealand Social Workers Registration Act, 3002, No 17. Retrieved from http://www.legislation.govt.nz/act/ public/2003/0017

Nicol, J. (1987). Abortion services in New Zealand. [A report prepared for the women, children and family health programme to aid in planning and policy formulation]. Wellington, NZ: Ministry of Health.

Onwuegbuzie, A. J. \& Leech, N. L. (2007). Sampling designs in qualitative research: Making the sampling process more public. The Qualitative Report, 12(2), 238-254.

Onwuegbuzie, A. J., Slate, J. R., Leech, N. L. \& Collins, K. M. T. (2007). Conducting mixed analysis: A general typology. International Journal of Multiple Research Approaches, 1(1), 4-17.

Richdale, J. M. (2010). Lifting the veil of silence: Personal abortion narratives in New Zealand, 1919-1937. (Doctoral thesis, University of Auckland).

Silva, M., Ashton, T., \& McNeill, R. (2011). Improving termination of pregnancy services in New Zealand. The New Zealand Medical Journal, 124(1339), 83-90.
Sparrow, M. (2010). Abortion then and now: New Zealand abortion stories from 1940 to 1980 . Wellington, NZ: Victoria University Press.

Statistics New Zealand. (2015). New Zealand in profile: An overview of New Zealand's people, economy and environment. Wellington, NZ: Author.

SurveyMonkey Inc. (n.d.). Palo Alto, California, USA. Retrieved from www.surveymonkey.com

Whitehead, A., \& Fanslow, J. (2005). Prevalence of family violence amongst women attending an abortion clinic in New Zealand. Australian and New Zealand Journal of Obstetrics and Gynaecology, 45, 321-324. 